\title{
Emergency hormonal contraception usage in genitourinary medicine clinic attenders
}

\author{
J K Evans, A Holmes, M Browning, G E Forster
}

Objective: To assess the indications for usage of emergency hormonal contraception amongst a population of London genitourinary medicine clinic attenders.

Methods: In a prospective study, 150 consecutive women receiving emergency hormonal contraception (EHC) were enrolled. The attending doctor completed a questionnaire of patient details and prescribed EHC with prophylactic prochlorperazine. Follow-up was arranged three weeks later, at which time outcomes and side-effects of therapy were recorded. For those women who did not reattend as planned case notes were reviewed at three months.

Results: Of 150 women surveyed, $100(66 \%)$ reported contraceptive method failure, 48 (32\%) had used no contraception at the time of last sexual intercourse and two requested EHC after sexual assault. Ninety three $(62 \%)$ reported condom failure, $7(5 \%)$ oral contraceptive pill failure. Seventy five (50\%) had used EHC before (range 1-10 times). Seventy one (47\%) women reattended within three months. Five $(3.3 \%)$ of the 150 women were pregnant; none of these cases had experienced nausea or vomiting whilst taking EHC. Side-effects were reported by $22(31 \%)$ of the 71 patients who reattended. Nine $(6 \%)$ women had been followed-up in the family planning advisory clinic. Of the 71 women who reattended, 39 (55\%) reported that their preferred future method of contraception would be condoms. Of the 150 women $19(13 \%)$ underwent tests for sexually transmissible infections within one month of presentation.

Conclusions: EHC usage in this population was associated with a failure rate of at least $3.3 \%$ and an overall side effect rate of $31 \%$. Despite requests for emergency contraception because of condom failure many elected to continue using condoms as their preferred method of contraception. The majority of women (53\%) did not return for follow-up or family planning advice, and so we believe that future contraceptive plans must be addressed at the time EHC is prescribed.

(Genitourin Med 1996;72:217-219)

Keywords: contraception; emergency

\section{Introduction}

The Chief Medical Officer's annual report of 1990 estimated that almost half of all conceptions were in some sense unwanted or unintended. "The Health of the Nation", published in 1992, highlighted sexual health as a key area and one of its stated objectives was to reduce the number of unwanted pregnancies. However, in 1992, 160,495 pregnancies were terminated in England and Wales. ${ }^{\prime}$

A recent survey found that $93 \%$ of women requesting abortion would have preferred to use emergency contraception to prevent an unplanned pregnancy. ${ }^{2}$ One study has shown that $40 \%$ of women attending for termination of pregnancy had heard of emergency contraception but did not know where to get it, and another $30 \%$ were unaware of emergency contraception as an option. ${ }^{3}$ A further study concluded that only $14 \%$ of women knew the correct time interval following sexual intercourse when the so-called "morning after pill" could be used. ${ }^{4}$

Emergency contraception may be obtained from general practitioners, some Accident and Emergency departments and family planning clinics. It has been reported that $98 \%$ of general practitioners who responded to a survey were willing to prescribe emergency contraceptive pills and $45 \%$ of Accident and Emergency departments reported prescribing it often. ${ }^{5}$
Increasingly, genitourinary medicine clinics are providing an integrated sexual health service including family planning/contraception and emergency hormonal contraception (EHC). The open access, self referral system operated by genitourinary medicine clinics makes them ideally placed to provide EHC. Between 1 January and 31 June, 1993, EHC was prescribed to 53 women at our clinic; over the first six months of 1994,141 women received $\mathrm{EHC}$. In view of this increasing trend and the fact that there had been no studies of emergency contraception usage among this population of women we instituted the following survey. The aim of this study was to examine the indications, usage and outcomes for women prescribed EHC in our genitourinary medicine clinic.

\section{Methods}

A prospective study of 150 consecutive women who were prescribed emergency hormonal contraception EHC, $(50 \mu \mathrm{g}$ ethinyloestradiol plus $500 \mu \mathrm{g}$ norgestrel) at our centre was carried out. Entry criteria were: (1) the woman should have had unprotected sexual intercourse no more than 72 hours prior to presentation, (2) she should have had no other episodes of unprotected sexual intercourse since her last menstrual period and (3) she should have no contraindications to treat- 
ment. ${ }^{6}$ These criteria for EHC prescription were based on the history given by the woman; no pregnancy tests were performed routinely. The attending physician completed a questionnaire giving details of the woman's reason for requesting EHC, parity, previous EHC usage and contraceptive plans. EHC was prescribed together with two doses of $5 \mathrm{mg}$ prochlorperazine to be taken thirty minutes before each set of tablets. The woman was given an information leaflet and an appointment to return to our joint Genitourinary medicine and family planning advisory clinic three weeks later. At follow-up a second questionnaire detailing side-effects and pregnancy was completed. In those cases where the woman did not return as planned, the notes were reviewed three months later. No recall procedure was instituted.

\section{Results}

The age range of the 150 women studied was 14-43 years (median 21 ). Seventy five (50\%) of the women reported using EHC before (median once, range 1-10). Twenty four $(16 \%)$ of the women had previous full term pregnancies and $34(23 \%)$ had undergone termination of pregnancy (median once, range $1-3)$. Of the 150 women surveyed, $100(66 \%)$ reported failure of contraceptive method. Forty eight $(32 \%)$ had used no contraception and two women attended after sexual assault. The median age of those women who had not used contraception was not significantly different from the group as a whole. Of 150 women $93(62 \%)$ reported condom failure and seven (5\%) of 150 women recognised potential failure of their oral contraceptive pill (such as concurrent use of broad spectrum antibiotics or forgotten pills). Seventy five $(50 \%)$ of the women stated that their last sexual intercourse had been with a regular partner ( 25 of whom reported using no contraception at the time of last sexual intercourse), $11(7 \%)$ with a casual partner and in $64(43 \%)$ of cases this information was not documented. Seventy eight of the women had received EHC within 24 hours of unprotected sexual intercourse, 35 between 25 and 48 hours, and 37 between 49 and 72 hours.

Fifty (33\%) of 150 women attended for follow-up within one month of initial presentation, of whom $9(6 \%)$ attended the family planning advisory clinic, as planned. A further $21(14 \%)$ of the women returned to the clinic between one and three months. Five of the 71 women who returned were pregnant $(3.3 \%$ of the total prescribed EHC); none of these cases experienced vomiting whilst taking EHC. Two of the pregnant women had been using no contraception and two were at the mid point of their menstrual cycle when given EHC. Side-effects were reported by $22(31 \%)$ of 71 women (10 nausea and vomiting, nine nausea, five abdominal pain, one breast tenderness and one described a panic attack). Twenty four women stated that the period occurring after taking EHC was abnormal, (six reported increased menstrual flow, five decreased flow,
14 an early period and four a delayed period). Women said that their preferred future method of contraception would be condoms in 39 cases $(51 \%)$, the oral contraceptive pill in 21 cases $(14 \%)$, the diaphragm in one case, the Depoprovera injection in one case and in seven cases $(10 \%)$ the woman was undecided or considering starting a family.

Nineteen (13\%) of 150 women underwent screening for sexually transmissible infections within one month of unprotected sexual intercourse; no new infections were diagnosed.

\section{Discussion}

This study demonstrates an EHC failure rate of at least 3.3 per 100 women per cycle which is comparable with failure rates documented in other populations. ${ }^{78}$ This may be an underestimate of the true rate in this group of women as we cannot assume that all of those who defaulted from follow-up were not pregnant. We would recommend routine prescription of an antiemetic with EHC as our study shows a reduction in nausea and vomiting from an expected $50 \%^{79}$ side effect rate to $27 \%$. No dystonic reactions to prochlorperazine were described.

It is of some concern that one third of women reported using no method of contraception at the time of last sexual intercourse, and that a third of women in stable relationships who requested EHC used no contraception. Six of the 150 women returned for a second prescription of EHC within three months. It is of interest that another six of the 150 volunteered that they had stopped using the oral contraceptive pill within the last month; this finding has been noted in other studies. $^{3}$

Compliance with follow-up was poor amongst this group of women although comparable with women using Accident and Emergency departments to obtain EHC. ${ }^{10}$ More successful follow-up has been reported amongst family planning clinic attenders using EHC. ${ }^{11}$ We were surprised that so many women elected to continue using condoms as their only method of contraception despite earlier condom failure. Recent health education promoting safer sex may account for this finding. Only $6 \%$ of the women studied attended the family planning advisory clinic at our centre and so, perhaps, it is not surprising that women returned requesting $\mathrm{EHC}$ again. A recent survey of family planning practice has shown that some clinics provide a woman with the oral contraceptive pill at her first attendance when requesting EHC. ${ }^{12}$ We were disappointed that so few women were screened for sexually transmitted infection despite giving a history of unprotected sexual intercourse. However, many women may not have perceived themselves to be at risk of sexually transmitted infection as at least half reported being in stable relationships. The clinician may have felt it inappropriate to offer such a screen at first presentation being unaware of the high default rate.

As our study has shown that compliance 
with follow-up is poor, we believe that future contraceptive plans and screening for sexually transmitted infection must be considered at the woman's first presentation as this may be the only opportunity available to health care professionals. A recent study of women attending a London genitourinary medicine clinic demonstrated that immediate provision of contraceptive education, advice and supplies is welcomed by clients. ${ }^{13}$ We hope that improved contraception services, including the appointment of a dedicated family planning nurse, will help to achieve these objectives at our centre.

We thank the nursing staff of the Ambrose King Centre and $\mathrm{Mr}$ $\mathrm{D}$ Webb, HIV Clinical Pharmacist, for their help in collecting the data.

1 Abortions statistics, OPCS monitor, 1992, series FMI, AB $94 / 1$.

2 Bromham DR, Cartmill RSV. Knowledge and usage of secondary contraception among patients requesting termination of pregnancy. $B M \mathcal{F}, 1993 ; 306: 556-7$.

3 Duncan G, Harper C, Mant D, Buchan $H$, Jones L. Termination of pregnancy: lessons for prevention. $B r \dot{F}$
Family Planning, 1990;15:112-7.

4 George J, Turner J, Cooke E, et al. Women's knowledge of emergency contraception. Br $\mathcal{F}$ Gen Pract 1994;44:451-4.

5 Walsh J. Policies and practices in postcoital contraceptive provision: a survey of general practitioners and hospital accident and emergency departments. $\mathrm{Br} f$ Family Planning, 1995;20:121-5.

6 National Association of Family Planning Doctors. Emergency contraception, guidelines for doctors. $\mathrm{Br} \mathcal{F}$ Emergency contraception, guidelines for doctors. $B r \mathcal{F}$
Family Planning 1992;18:pull-out section.

7 Yuzpe AA, Percival Smith R, Rademaker AW. A multicentre clinical investigation employing ethinyloestradiol combined with dl-norgestrel as a post coital contraceptive agent. Fertil Steril 1982:37:508-13.

8 Guillebaud J, Kubba AA, Rowlands S, et al. Postcoital contraception with danazol compared with an ethinyloestradiol-norgestrel combination or insertion of intra-uterine device. F Obstet Gynaecol 1993;3(suppl 2):s64-8.

9 Percival-Smith R, Ross A. Postcoital contraception using dl-norgestrel-ethinyloestradiol combination. Contraception 1978;17:247-52

10 Roberts RN, Moohan NN, McNeill S, Lyons MS. Audit of an emergency contraceptive service. Br $\mathcal{F}$ Family Planning 1995;21:22-4.

11 Bagshaw SN, Edwards D, Tucker AK. Ethinyl oestradiol and d-norgestrel is an effective emergency postcoital contraceptive. NZ F Obstet Gynaecol 1988;28:137-40.

12 Webb A, Morris J. Practice of postcoital contraceptionthe results of a national survey. $B r f$ Family Planning 1993;18:113-8.

13 Masters L, Nicholas H, Bunting P, Welch J. Family Planning in genitourinary medicine: an opportunistic service? Genitourin Med 1995;71:103-5. 